\title{
Excess hard X-ray emission from the obscured low luminosity AGN in the nearby galaxy M 51 (NGC 5194)
}

\author{
Y. Fukazawa ${ }^{1,3}$, N. Iyomoto ${ }^{2,3}$, A. Kubota ${ }^{3}$, Y. Matsumoto ${ }^{3}$, and K. Makishima ${ }^{3}$ \\ 1 Department of Physical Sciences, Graduate School of Science, Hiroshima University, 1-3-1 Kagamiyama, \\ Higashi-Hiroshima, Hiroshima 739-8526, Japan \\ 2 Institute of Space and Astronautical Science, 3-1-1 Yoshinodai, Sagamihara, Kanagawa 229-8510, Japan \\ 3 Department of Physics, Graduate School of Science, University of Tokyo, 7-3-1 Hongo, Bunkyo-ku, \\ Tokyo 113-0033, Japan
}

Received 29 January 2001 / Accepted 27 April 2001

\begin{abstract}
We observed the nearby galaxy M 51 (NGC 5194) with BeppoSAX. The X-ray properties of the nucleus below $10 \mathrm{keV}$ are almost the same as the ASCA results regarding the hard component and the neutral Fe K $\alpha$ line, but the intensity is about half of the ASCA 1993 data. Beyond this, in the BeppoSAX PDS data, we detected a bright hard X-ray emission component which dominates above $10 \mathrm{keV}$. The 10-100 keV flux and luminosity of this component are respectively $2 \times 10^{-11} \mathrm{erg} \mathrm{s}^{-1} \mathrm{~cm}^{-2}$ and $2 \times 10^{41} \mathrm{erg} \mathrm{s}^{-1}$. These are about 10 times higher than the extrapolation from the soft X-ray band, and similar to the flux observed with Ginga, which found a bright power law component in 2-20 keV band. Considering other wavelength properties and the X-ray luminosity, together with strong neutral Fe K line, the hard X-ray emission most likely arises from a low luminosity active nucleus, which is obscured with a column density of $\sim 10^{24} \mathrm{~cm}^{-2}$. This suggests that hidden low luminosity AGNs may well be present in other nearby galaxies. We interpret the discrepancy between Ginga and other X-ray satellites to be due to a large variability of absorption column density toward the line of sight over several years, suggesting that the Compton thick absorption material may be present on a spatial scale of a parsec. Apart from the nucleus, several ultra-luminous off-nuclear X-ray sources detected in M 51 exhibit long-term time variability, suggesting the state transition similar to that observed in Galactic black hole candidates.
\end{abstract}

Key words. galaxies: active - galaxies: nuclei - galaxies: individual: M 51

\section{Introduction}

M 51 is a face-on spiral galaxy, also known as the Whirlpool galaxy; it is located at a distance of $9.6 \mathrm{Mpc}$ (Sandage \& Tammann 1975). Optical studies of emission lines classified it as a LINER (Low Ionization Nuclear Emission Region) or a Seyfert 2 galaxy (Stauffer 1982; Filippenko \& Sargent 1985; Ho et al. 1997b). Furthermore, Ho et al. (1997c) suggested the presence of a broad $\mathrm{H} \alpha$ line. Kohno et al. (1996) found a nuclear molecular disk, and constrained the dynamical mass within $70 \mathrm{pc}$ of the nucleus to be $(4-7) \times 10^{6} M_{\odot}$. This indicates that M 51 hosts as massive a black hole as many bright AGNs.

Observations in other wavelengths also strongly suggest the presence of an AGN in M 51, although previous X-ray observations of M 51 gave puzzling results. Einstein (Palumbo et al. 1985) and ROSAT (Marston et al. 1995;

Send offprint requests to: Y. Fukazawa,

e-mail: fukazawa@hirax6.hepl.hiroshima-u.ac.jp
Ehle et al. 1995) observations constrained the soft X-ray luminosity of the M 51 nucleus to be $L_{\mathrm{X}}<5 \times 10^{39} \mathrm{erg} \mathrm{s}^{-1}$. Ginga scanning observations in 1988 detected bright hard X-ray emission with $L_{\mathrm{X}}=1.2 \times 10^{41} \mathrm{erg} / \mathrm{s}(2-20 \mathrm{keV})$ with a photon index of $1.43 \pm 0.08$ (Makishima et al. 1990) and an intrinsic absorption of $<7 \times 10^{21} \mathrm{~cm}^{-2}$. Such hard nuclear X-ray emission is often considered to be evidence of Low Luminosity AGN (LLAGN), but it should have been detected with Einstein and ROSAT if the flux did not change significantly. After the Ginga observations, ASCA observed M 51 in the hard X-ray band $(2-10 \mathrm{keV})$ in 1993 , and did not detect such a bright hard component (Terashima et al. 1998a). Instead, a faint hard $\mathrm{X}$-ray continuum with a neutral Fe $\mathrm{K}$ line was detected, whose flux was an order of magnitude lower than that measured by Ginga. One possibility is that the difference between these observations is due to time variability with a large amplitude. Such a strong time variability is not often observed for low luminosity AGNs, and therefore is 

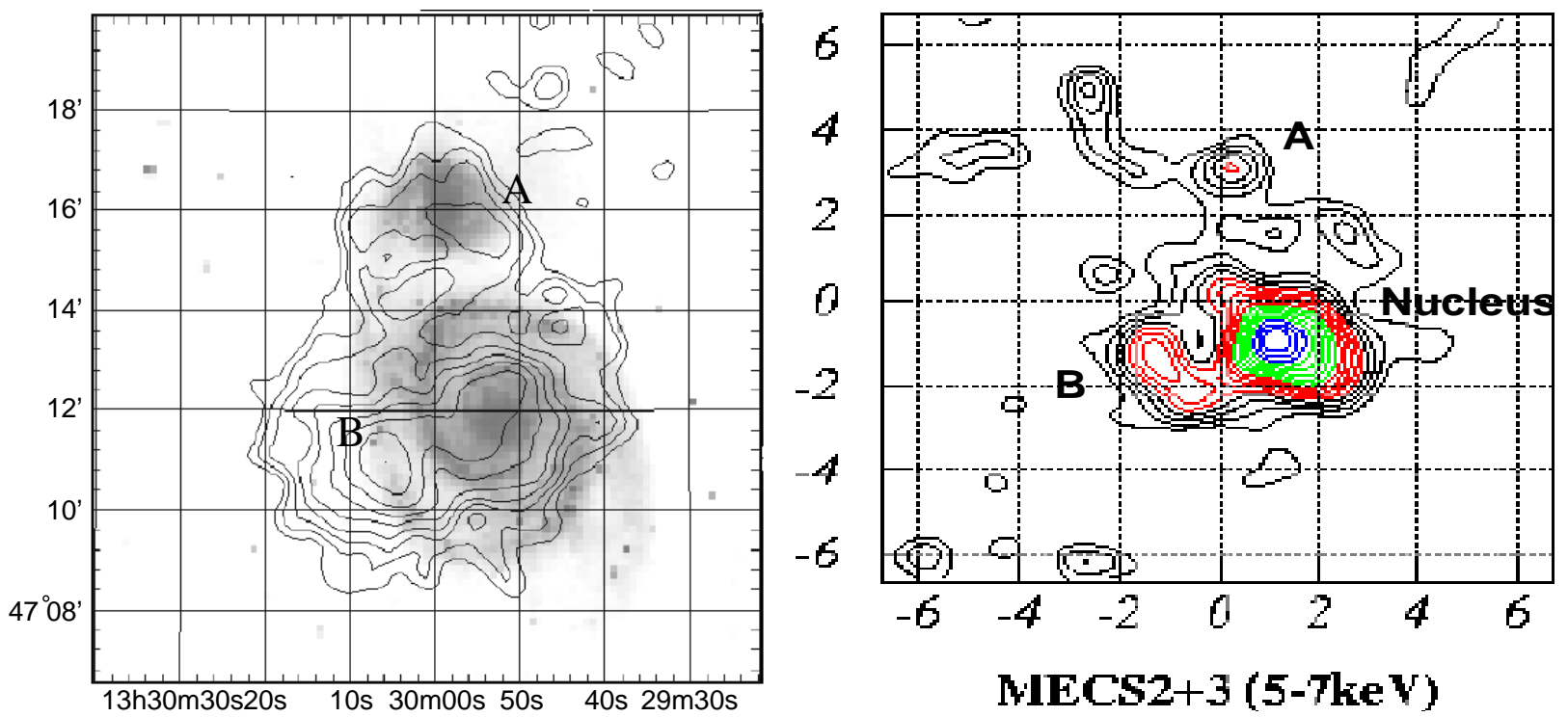

Fig. 1. BeppoSAX MECS images of M 51. a) MECS image (contour) in the total band overlaid on the optical image (gray scale). b) MECS image in the energy band of $5-7 \mathrm{keV}$. The center position is (13h $30 \mathrm{~m} 00 \mathrm{~s}, 47 \mathrm{~d} 13 \mathrm{~m} 00 \mathrm{~s})$. The axis unit is arcmin.

quite interesting if it is correct. The other possibility is a strong change of the intrinsic absorption. The key phenomenon that can help in resolving this mystery is the presence of a neutral Fe K line emission detected by ASCA (Terashima et al. 1998a), which indicates that during the ASCA observations, the nucleus of M 51 was heavily obscured by Compton thick material below $10 \mathrm{keV}$, as found in some Seyfert 2 galaxies (e.g. Risaliti et al. 1999). This idea can be confirmed by X-ray observations at energies above $10 \mathrm{keV}$.

Detection of an obscured hard X-ray emission from M 51 would suggest that many galaxies may harbor previously unknown hidden LLAGNs emitting below $10 \mathrm{keV}$. Moreover, such a detection can be valuable for a study of the physical structure of Compton thick absorbing material around AGNs. To further investigate such a possibility, we performed an X-ray observation of M 51 with BeppoSAX, sensitive to hard X-ray emission (Boella et al. 1997b).

\section{Observations and data reduction}

We observed M 51 with BeppoSAX on Jan. 18-20, 2000. The three Narrow Field Instruments, LECS (Parmar et al. 1997), MECS2+3 (Boella et al. 1997b), and PDS (Frontera et al. 1997) collected the data during the observation. We obtained the net exposures of $37 \mathrm{ks}, 97 \mathrm{ks}$, and $45 \mathrm{ks}$, for LECS, MECS, and PDS, respectively. We utilized the cleaned and linearized data supplied by the BeppoSAX Science Data Center. The light curves, spectra, and images were reduced with the standard software, such as XSELECT and FTOOLS-4.2. Throughout this paper, the data of two MECS instruments were summed together. The cross-normalization constant between MECS and PDS is set to be 0.85 in this paper (Fiore et al. 1999).
In addition, we utilized ASCA (Tanaka et al. 1994) archival data in order to investigate the time variability of the M 51 nucleus. ASCA observed M 51 twice, on May 1113, 1993 and May 5-7, 1994. The results of ASCA 1993 observation were reported by Terashima et al. (1998a). Ptak et al. (1999) also performed the analysis of both 1993 and 1994 data, but they did not consider the contamination by the neighboring off-nuclear bright X-ray source. The screened data were obtained from the ASCA archival data center. In the data reduction, we imposed the selection criterion on the GIS data requiring that the rigidity is $>6 \mathrm{GeV} \mathrm{c}^{-1}$ (Ohashi et al. 1996; Makishima et al. 1996). The net GIS exposure is $35-38 \mathrm{ksec}$ for both observations, but it was only $12 \mathrm{ksec}$ for the SIS data in 1994 due to problems of the onboard data processing.

\section{Image analysis}

Before spectral analysis can be performed on the nuclear component, it is necessary to investigate $\mathrm{X}$-ray images because M 51 is known to contain several non-nuclear X-ray sources. In Fig. 1, we show the X-ray images taken with the BeppoSAX MECS. The MECS image in the total band is overlaid on the optical image taken from SkyVIEW from NASA/GSFC. Three X-ray sources are clearly visible: the nucleus of $\mathrm{M} 51$, a source in the companion galaxy NGC 5195 (source A), and a source to the east of the nucleus (source B). The nucleus is particularly bright in the $5.7-7.0 \mathrm{keV}$ band, where a neutral Fe $\mathrm{K}$ line exists. Recently, Terashima et al. (2001) confirmed that this source is resolved as a nuclear point source with Chandra. The source B is not the supernova SN1994I, since its position is different and the flux observed in ROSAT is quite low (Immler et al. 1998).

In Fig. 2, we show ASCA GIS images of M 51 taken in 1993 and 1994. The nucleus and the source A are always 


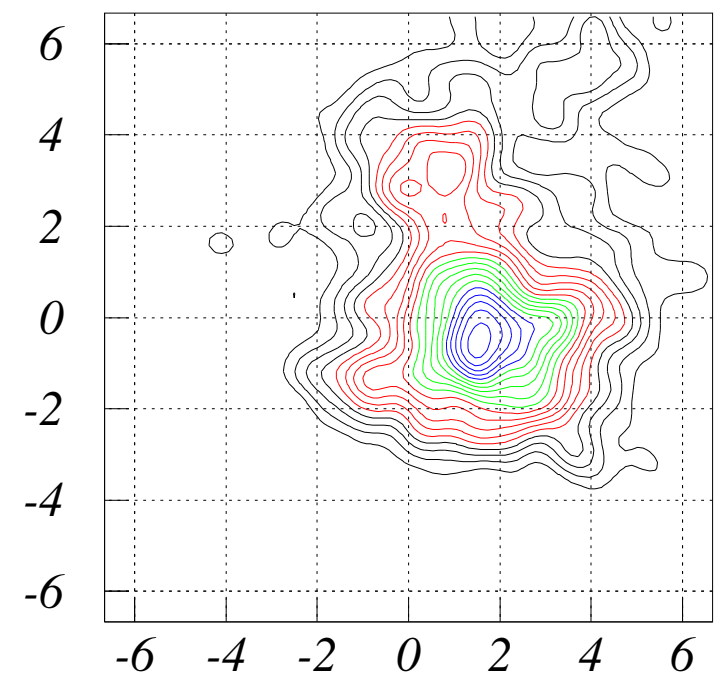

GIS2+3 1993 (0.7-10keV)

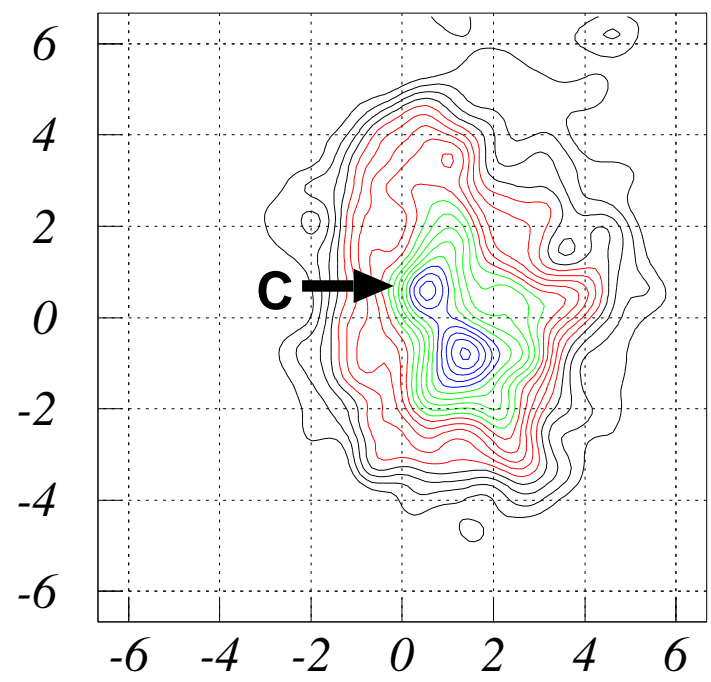

GIS2+3 1994 (0.7-10keV)

Fig. 2. ASCA GIS images of M 51 in 1993 (left) and 1994 (right). The axis unit is arcmin.

visible, in the ASCA 1993 and BeppoSAX 2000 observations. The source B is not seen in the ASCA GIS images, indicating the time variability of this source. On the other hand, the north-east X-ray source (source C) that was visible in the ASCA 1994 observation is not seen in the ASCA 1993 and BeppoSAX image.

Sources $\mathrm{B}$ and $\mathrm{C}$ are thought to correspond to the ROSAT PSPC point sources R3 and R1 in Marston et al. (1995), respectively. All the detected sources have X-ray luminosities in the range of $\sim 10^{39-40} \mathrm{erg} \mathrm{s}^{-1}$.

\section{Spectral analysis}

\subsection{BeppoSAX spectra of the nucleus}

In order to investigate the spectrum of the nucleus, we extracted data from the imaging instruments onboard BeppoSAX corresponding to a radius of $2^{\prime}$ so that neighboring X-ray sources do not contaminate the spectra of the nuclear source. The background spectra were prepared using data from the same detector region, utilizing the standard background data set (Nov. 1998 for MECS, Dec. 1999 for LECS), and we subtracted them from the on-source spectra. In the spectral fitting, we used the standard response matrices released in September 1997.

In Fig. 3, we show the X-ray spectra of LECS and MECS. The data cannot be fit by a simple power law; they reveal both Fe-L and Fe $\mathrm{K}$ line features, but the Raymond-Smith thermal model (Raymond \& Smith 1977) with the Galactic absorption of $1.5 \times 10^{20} \mathrm{~cm}^{-2}$ (Stark et al. 1992) does not fit the data either: the reduced chi-square is $\sim 2$ for both models. These spectral features are similar to the ASCA 1993 spectra. Thus, we fit the spectra with a model including Raymond-Smith thermal plasma plus a power law, and including an additional narrow line. The metal abundances are fixed to $0.2 \times$ Solar with the Solar metal abundance ratios

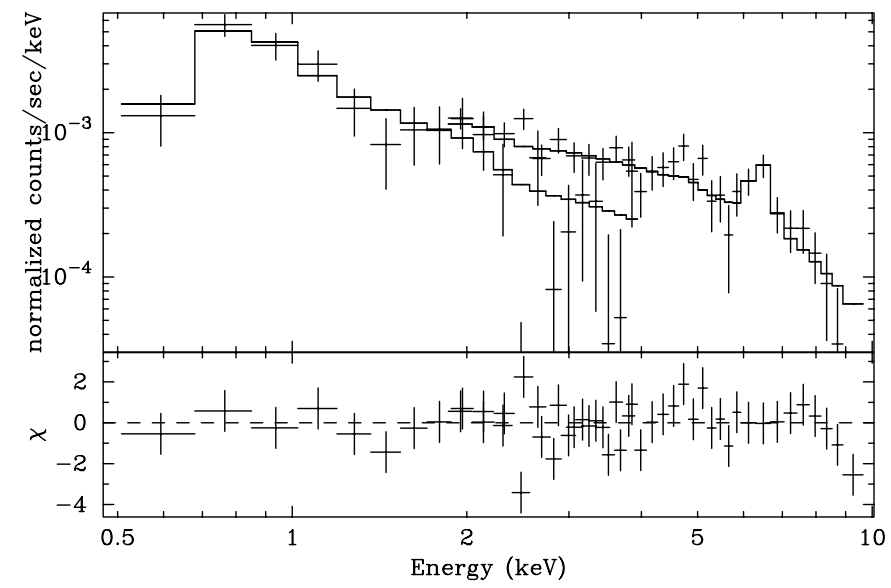

Fig. 3. LECS and MECS spectra of the nucleus. The solid line represents the best-fit model (Raymond-Smith model + power law model + Gaussian).

(Anders \& Grevesse 1989). Such a model was previously applied to the ASCA 1993 spectra (Terashima et al. 1998a). The fit is acceptable as shown in Fig. 3 and Table 1. The power law photon index of $1.59 \pm 0.23$ is not well constrained, but it is consistent with the ASCA results. The Fe $\mathrm{K}$ line center energy is $6.47 \pm 0.14 \mathrm{keV}$, indicating that it is due to fluorescence from the neutral iron, in agreement with the ASCA results. The equivalent width of the $\mathrm{Fe} \mathrm{K}$ line is $0.9_{-0.5}^{+0.4} \mathrm{keV}$, which is within the error range of the 1993 ASCA results. The temperature of the Raymond-Smith plasma is $0.51 \pm 0.16 \mathrm{keV}$. The flux and luminosity of the power law component are $3.4_{-0.3}^{+0.4} \times 10^{-13} \mathrm{erg} \mathrm{s}^{-1} \mathrm{~cm}^{-2}$ and $3.6_{-0.3}^{+0.4} \times 10^{39} \mathrm{erg} \mathrm{s}^{-1}$, respectively in $2-10 \mathrm{keV}$ band, about $30 \%$ of 1993 ASCA results. The strength of $\mathrm{Fe} \mathrm{K}$ line is also smaller than that of 1993 ASCA value of $(4-14) \times 10^{-6} \mathrm{c} \mathrm{s}^{-1} \mathrm{~cm}^{-2}$.

We conclude that the spectral properties below $10 \mathrm{keV}$ measured in the BeppoSAX data are almost the same as 

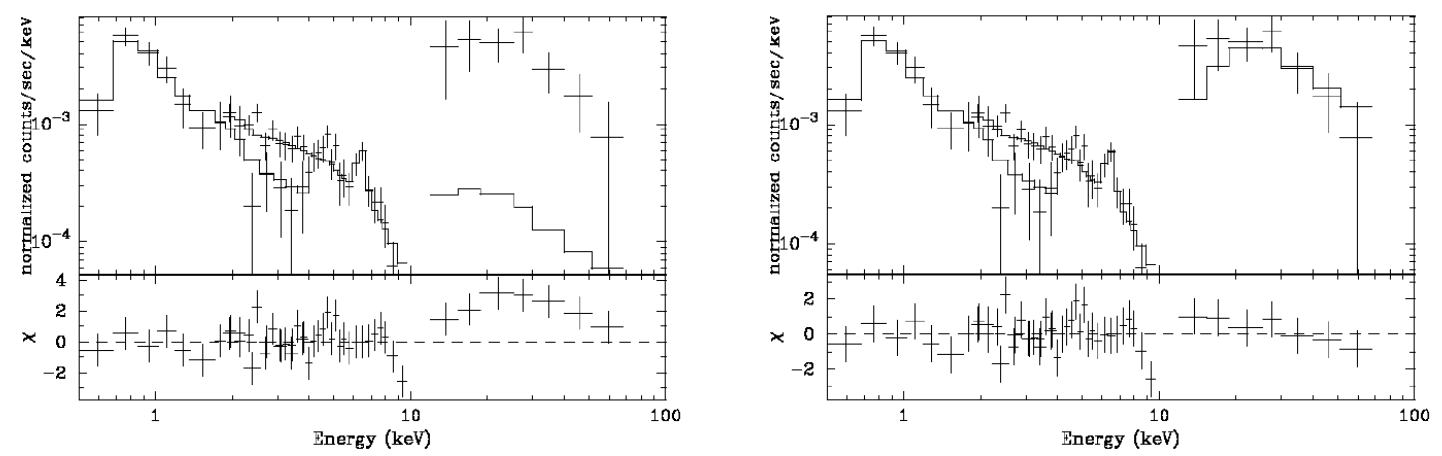

Fig. 4. LECS, MECS, and PDS spectra of the nucleus. The solid line represents the best-fit model (the same as Fig. 4) for the LECS and MECS spectra (left), and the best-fit model (Raymond-Smith model + power law model + Gaussian + absorbed power law) for all the instruments (right).

the ASCA results except for the flux. To extend the bandpass beyond that of the imaging instruments $(10 \mathrm{keV})$, we plot a PDS spectrum together with the LECS and MECS spectra in Fig. 4a. The solid line represents the best-fit model obtained by the LECS and MECS spectral analysis. It can be seen that the PDS flux is higher by a factor of $\sim 10$ compared with the extrapolation of the best-fit model of LECS and MECS, and significant photon flux is detected up to $50 \mathrm{keV}$. This spectral feature is quite similar to that seen in Compton-thick Seyfert 2 galaxies such as NGC 4945 (Done et al. 1996), Circinus Galaxy (Matt et al. 1999), and Mrk 3 (Cappi et al. 1999).

We add an absorbed power law component to represent this hard X-ray emission, and fit the LECS + MECS + PDS spectra simultaneously. The best-fit results are shown in Table 1 and Fig. 4b. The wide-band $\mathrm{X}$-ray spectra require a hard X-ray component strongly absorbed by optically thick material with a column density $\sim 10^{24} \mathrm{~cm}^{-2}$. The best-fit parameters other than this newly included component do not change significantly. Since the photon index and absorption column density for the hard X-ray component are not well constrained separately (as they are correlated in spectral fits), to estimate the absorbing column we fixed the photon index at 1.9, typical for Seyfert 1 galaxies without a reflection component. Then, the absorption column density becomes $5.6_{-1.6}^{+4.0} \times 10^{24} \mathrm{~cm}^{-2}$, and the X-ray flux and luminosity are $1.9 \times 10^{-11} \mathrm{erg} \mathrm{s}^{-1} \mathrm{~cm}^{-2}$ and $2.0 \times 10^{41} \mathrm{erg} \mathrm{s}^{-1}$, respectively in $10-100 \mathrm{keV}$ band. The absorption - corrected X-ray flux is $2.2 \times 10^{-11} \mathrm{erg} \mathrm{s}^{-1} \mathrm{~cm}^{-2}$ in $2-20 \mathrm{keV}$ band, similar to the Ginga results, and $1.5 \times 10^{-11} \mathrm{erg} \mathrm{s}^{-1} \mathrm{~cm}^{-2}$ in $2-10 \mathrm{keV}$ which should be easily detected with ASCA if no absorption was present. When we fit only the PDS spectrum with an absorbed power law model by fixing the power law photon index to be 1.9 , we obtained only the upper limit of $N_{\mathrm{H}}<5.4 \times 10^{24} \mathrm{~cm}^{-2}$. This is most likely because the model adopted by us, with a luminous but heavily absorbed hard component plus a soft component, has an apparent absorption feature located in the energy band between MECS and PDS. To investigate a possible presence of a Compton reflection component, we include it by fitting the data to the spectral model "pexrav" as implemented in XSPEC; with this, the reflection fraction becomes $<18 \%$.

\subsection{ASCA spectra of the nucleus}

Since the result of the 1993 ASCA observation are reported in Terashima et al. (1998a), we here show the result of the 1994 observation. Since the exposure time of the SIS is short, we created only the GIS spectrum. The source $\mathrm{C}$ is located at $\sim 2^{\prime}$ from the nucleus. Thus we created spectra using counts detected only within $1.5^{\prime}$ of the nucleus, where the source $\mathrm{C}$ may contaminate the nuclear spectrum by $\sim 20 \%$. The spectral model used for the fits of the ASCA data is the same as that for LECS and MECS spectra: Raymond-Smith plasma, plus power law, and one emission line. The normalizations of thermal and power law components are left free, but the other model parameters are fixed to the values measured in the 1993 observation, due to the poor quality of 1994 data.

The spectrum and best-fit model are shown in Fig. 5, which tells us that the overall property of the spectrum apart from the normalization is almost the same as that of ASCA 1993 data and BeppoSAX data. The X-ray flux of the power law component is $\sim 3 \times 10^{-13} \mathrm{erg} \mathrm{s}^{-1} \mathrm{~cm}^{-2}$ in $2-10 \mathrm{keV}$ band, about $30 \%$ of that measured in the 1993 data and almost the same as BeppoSAX data. The details of the Fe $\mathrm{K}$ line are ambiguous due to the small extraction area. In order to constrain the Fe K line strength better, we extended the integration radius to $3.75^{\prime}$. Taking into account that about $45 \%$ of photons in this spectrum come from the source $\mathrm{C}$, we obtain an upper limit on the Fe K line strength of $2.7 \times 10^{-6}$ photons s$~^{-1} \mathrm{~cm}^{-2}$, significantly smaller than that measured in the 1993 ASCA data.

\subsection{Time variability of the nucleus}

We investigate the short time variability properties of the hard component of the nucleus. We make light curves in the $3-10 \mathrm{keV}$ band (MECS) and 10-100 keV band (PDS). The MECS light curve shows a counting rate from the region within $2^{\prime}$ of the nucleus without background subtraction, since the count rate of the background is only $25 \%$. 
Table 1. Results of joint spectral fittings of M 51 nucleus with various models.

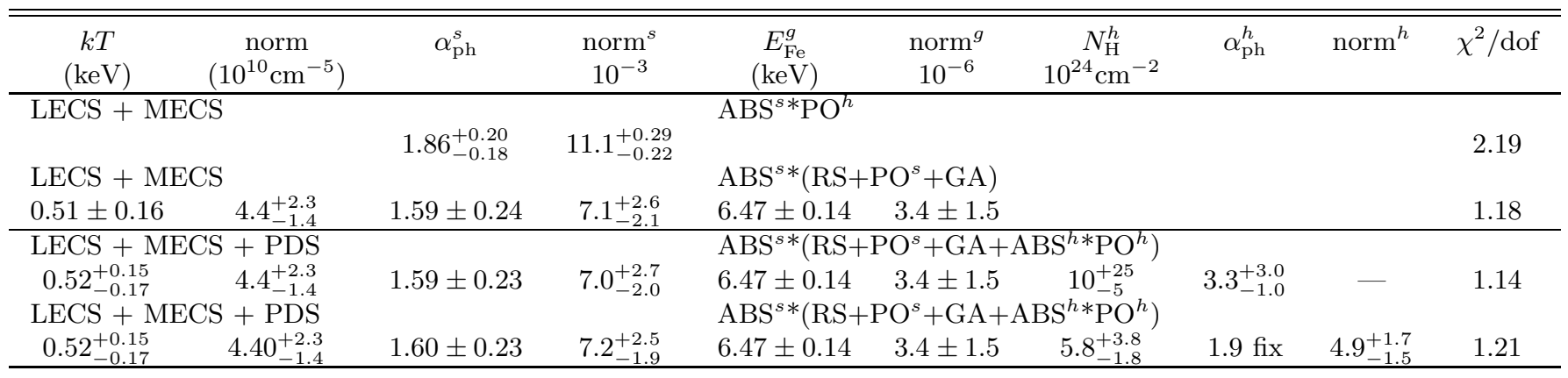

$\mathrm{ABS}^{s}$ and $\mathrm{ABS}^{h}$ : Photoelectric absorption of the Galactic and intrinsic material, RS: Raymond-Smith thermal model, $\mathrm{PO}^{s}$ and $\mathrm{PO}^{h}$ : Power law model, GA: Gaussian line.

$g$ : Parameters of a Gaussian model.

The unit of normalizations (norm) of power law and Gaussian is c s${ }^{-1} \mathrm{~cm}^{-2} \mathrm{keV}^{-1}$ at $1 \mathrm{keV}$ and c s${ }^{-1} \mathrm{~cm}^{-2}$, respectively.

The PDS light curve is created after background subtraction. We do not find any significant short-term time variability in either light curve.

In Fig. 6, we show the long-term time variability of the $\mathrm{X}$-ray flux of the power law component in the $2-10 \mathrm{keV}$ band and absorption-corrected $2-20 \mathrm{keV}$ band. The X-ray flux in $2-10 \mathrm{keV}$ band represents the scattered component of the nuclear emission, and in 1994, it drops to $30 \%$ of the 1993 value. Its flux in the 2000 BeppoSAX data is almost the same as that of 1994 ASCA observation. The absorption-corrected flux in $2-20 \mathrm{keV}$ of Ginga and BeppoSAX PDS corresponds to the direct nuclear emission. Nominally the 2000 BeppoSAX flux is twice the 1988 Ginga value, but the errors are large, and these fluxes are consistent with the values being the same. The Fe line equivalent width is constant within the error range as described in Sect. 4.2.

\subsection{Off-nuclear $X$-ray sources}

M 51 includes several off-nuclear point-like sources. The typical X-ray luminosity of the brightest X-ray sources is $10^{39-40} \mathrm{erg} \mathrm{s}^{-1}$, much higher than the Eddington limit for accreting neutron stars. In the ASCA and BeppoSAX observations, three off-nuclear sources were visible (Figs. 1 and 2). The source A is associated with NGC 5195, and its flux appears constant. The other two sources (B and C) are located in the spiral arms. They exhibit strong time variability, and are visible when they become as bright as the nucleus.

The source B shows a large time variability in 2000 as shown in Fig. 7, where the count rate in the second half of the observation decreases to $\sim 50 \%$ of that measured in the first half. This is the third example of short-term time variability of the off-nuclear ultra-luminous X-ray sources, after those in IC 342 (Okada et al. 1998; Kubota et al. 2001) and M 82 (Matsumoto et al. 1999). The spectra of the sources A and B are determined from the counts collected within $2^{\prime}$ of their position. For the source A,

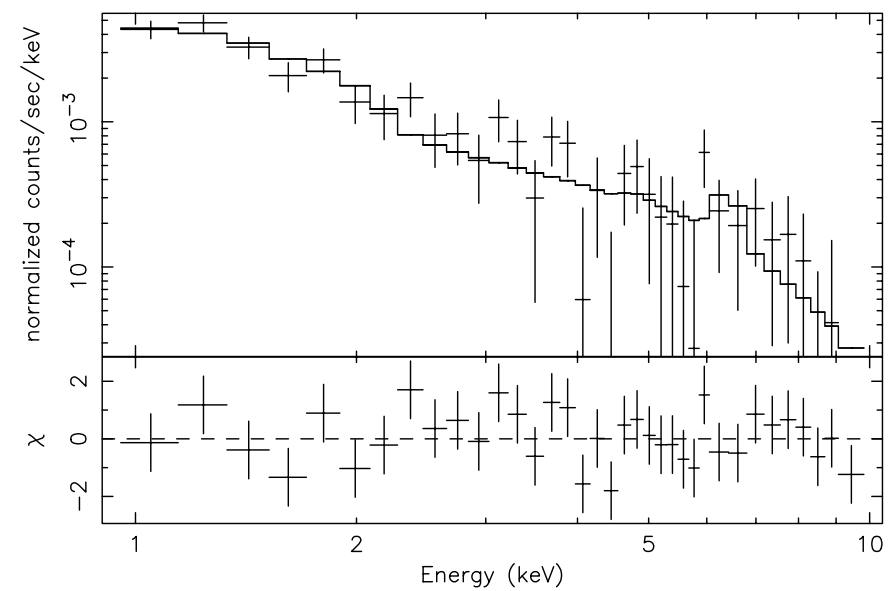

Fig. 5. ASCA GIS spectrum of the nucleus in 1994. The solid line represents the best-fit model (see text).

we prepared the spectra using the ASCA 1993 data and the BeppoSAX data. We do not use the ASCA 1994 data because the GIS instrument cannot resolve the source A clearly (see Fig. 2). The source B is only visible in the BeppoSAX data, and its count rate varies significantly. We thus analyzed two MECS spectra; one is integrated in the bright phase (0-110000 $\mathrm{s}$ in Fig. 7$)$, and the other is in the faint phase (110000-190000 s in Fig. 7).

The X-ray spectra of these ultra-luminous off-nuclear $\mathrm{X}$-ray sources are known to be generally described by multi-color disk black body (MCD: Mitsuda et al. 1984; Makishima et al. 1986; Mizuno et al. 1999; Makishima et al. 2000; Kotoku et al. 2001), and thus we try to fit the spectra by a power law model and MCD model. The spectrum of source $\mathrm{A}$ is almost the same between the 1993 ASCA and BeppoSAX observations, and thus we fitted both data sets simultaneously. We performed spectral fittings with the energy band above $2 \mathrm{keV}$, to avoid the uncertainty of the diffuse thermal emission due to the different angular resolution among instruments. Instead, we include the Raymond-Smith thermal model to represent the diffuse emission. Its temperature cannot be well 


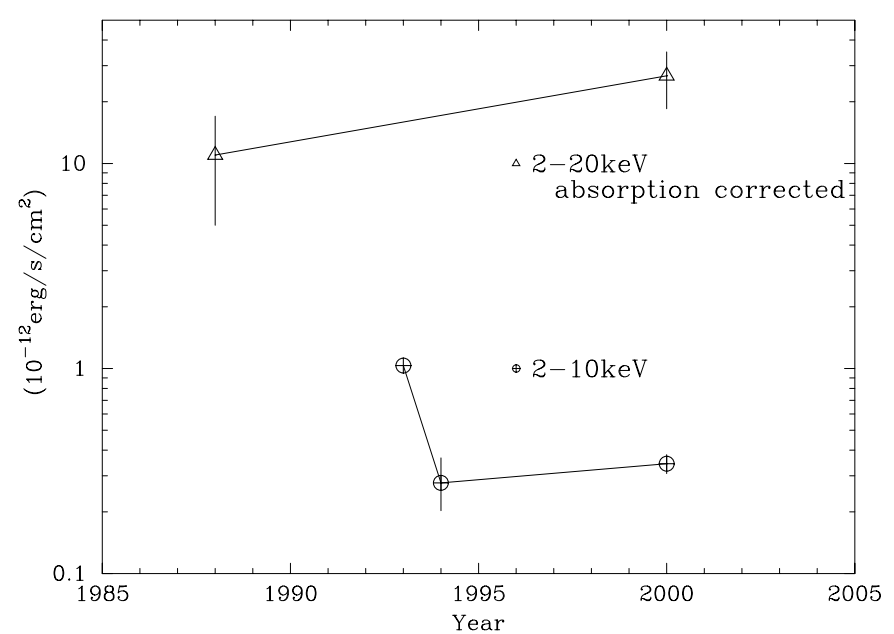

Fig. 6. Light curve of the M 51 nucleus measured by various satellites. The open circles represent the flux of the weak continuum in $2-10 \mathrm{keV}$ band, which are thought to be a scattered or reflected component. The open triangles are the flux of the bright nuclear direct continuum in $2-20 \mathrm{keV}$ band.

determined, and thus we fixed the temperature of Raymond-Smith model to 0.39 and $0.35 \mathrm{keV}$ for source $\mathrm{A}$ and $\mathrm{B}$, respectively, based on the spectral fitting of the ASCA SIS data around each source. Since we are interested in the hard component rather than soft thermal emission, we performed the spectral fitting above $2 \mathrm{keV}$. The results are summarized in Table 2 and Fig. 8.

The limited photon statistics does not allow us to distinguish between these two models for both sources. The inner disk temperature of MCD model for the source A, $1.5-2.2 \mathrm{keV}$, is typical for ultra-luminous off-nuclear X-ray sources in other galaxies. The luminosity of the MCD component in the $2-10 \mathrm{keV}$ band is $3.2 \times 10^{39} \mathrm{erg} \mathrm{s}^{-1}$ for source A. For the source B, the spectrum in the high flux period seems to be harder than that in the low flux period; the power law photon index or the temperature of the MCD model is significantly different. We expect a constant radius of the MCD model despite different temperatures, but that cannot be tested due to the poor data quality. The luminosities of the MCD component in the $2-10 \mathrm{keV}$ band of the source B are $3.8 \times 10^{39} \mathrm{erg} \mathrm{s}^{-1}$ and $1.2 \times 10^{39} \mathrm{erg} \mathrm{s}^{-1}$ for the bright and faint state, respectively.

In order to study the long-term time variability of these sources, we utilized the archival ROSAT HRI data from HEASARC (NASA/GSFC High Energy Astrophysics Science Archive Research Center) and the paper of Ehle et al. (1995). We cannot resolve the source A clearly in the HRI images, and thus the source A may be an assembly of discrete sources in NGC 5195. The constant flux is consistent with this scenario. The flux histories for the sources B and $\mathrm{C}$ are summarized in Table 3 . These are typically in the range of $(0-3) \times 10^{-13} \mathrm{erg} \mathrm{cm}^{-2} \mathrm{~s}^{-1}$, at a level comparable to the ASCA and BeppoSAX measurements. The source $\mathrm{B}$ varies by a factor of 2 , while the variability of the source $\mathrm{C}$ is quite strong, by a factor of $>5$.

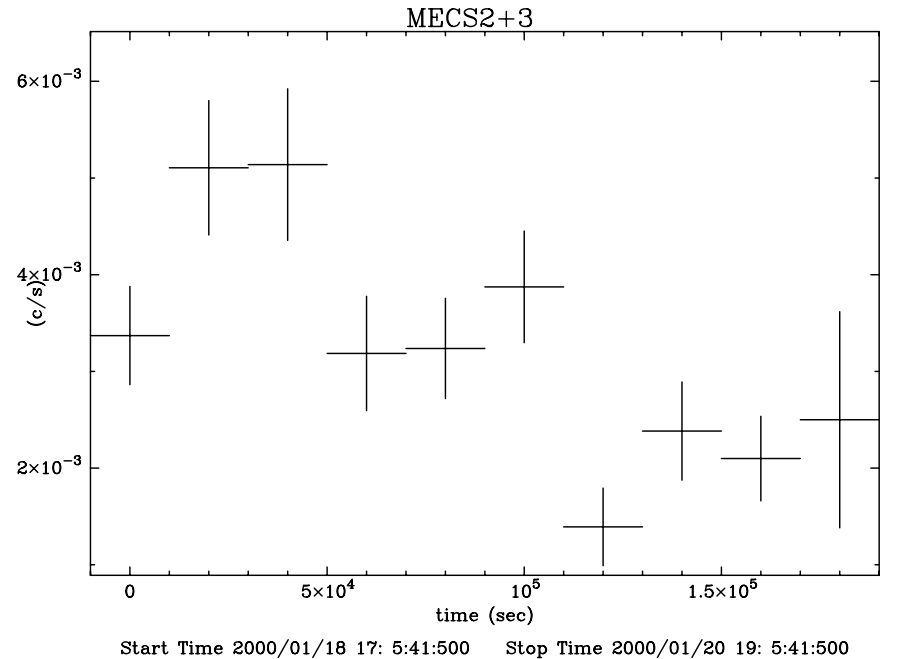

Fig. 7. MECS light curve of the off-nuclear source B.

\section{Discussion}

\subsection{Bright hard $X$-ray emission}

In our observations of M 51 with BeppoSAX, we detected in the PDS data bright hard X-ray emission above $10 \mathrm{keV}$, which we interpret as associated with an active nucleus present in the galaxy. This inference is supported by the fact that in the MECS data we detect a neutral Fe K line emission from the central point-like source, and such emission is consistent with the ASCA observations. The flux above $10 \mathrm{keV}$ is about 10 times higher than the extrapolation of the nuclear flux detected by the MECS below $10 \mathrm{keV}$. Considering these spectral features, we infer that M 51 harbors a heavily obscured Seyfert 2 type nucleus, with the column density $10^{24} \mathrm{~cm}^{-2}$.

The X-ray luminosity of this hard component is $2.0 \times 10^{41} \mathrm{ergs}^{-1}$ in the $10-100 \mathrm{keV}$ band, and thus M 51 is probably the first low-luminosity Compton thick Seyfert 2 AGN. The existence of AGN with X-ray luminosity of $\sim 10^{41} \mathrm{ergs}^{-1}$ is reasonable, according to the prediction from the correlation of $\mathrm{H} \alpha$ and X-ray luminosity in Seyfert 1 galaxies (Ward et al. 1988), and the correlation of $\left[\mathrm{O}_{\mathrm{III}}\right]$ and X-ray luminosity of Seyfert galaxies (Mulchaey et al. 1994), based on the M 51 value of $L_{\mathrm{OIII}} \sim L_{\mathrm{H} \alpha} \sim 10^{39} \mathrm{erg} \mathrm{s}^{-1}$. Moreover, the presence of an obscured nucleus in M 51 is suggested by a dense molecular disk with $>3 \times 10^{23} \mathrm{~cm}^{-2}$ detected around the nucleus by HCN and CO observations (Kohno et al. 1996).

Nevertheless, we cannot completely rule out the possibility that the PDS spectrum is contaminated by bright hard sources, because the absorption is ambiguous if one considers the PDS spectrum alone. However, there are several observational results which support the above conclusion. The Ginga observation - using the scanning mode, and thus allowing good positional accuracy - indicated that the bright hard X-ray emission indeed comes from the M 51 nucleus (Makishima et al. 1990). Since the hard X-ray flux is similar between the Ginga LAC and the BeppoSAX PDS, the hard emission detected from 

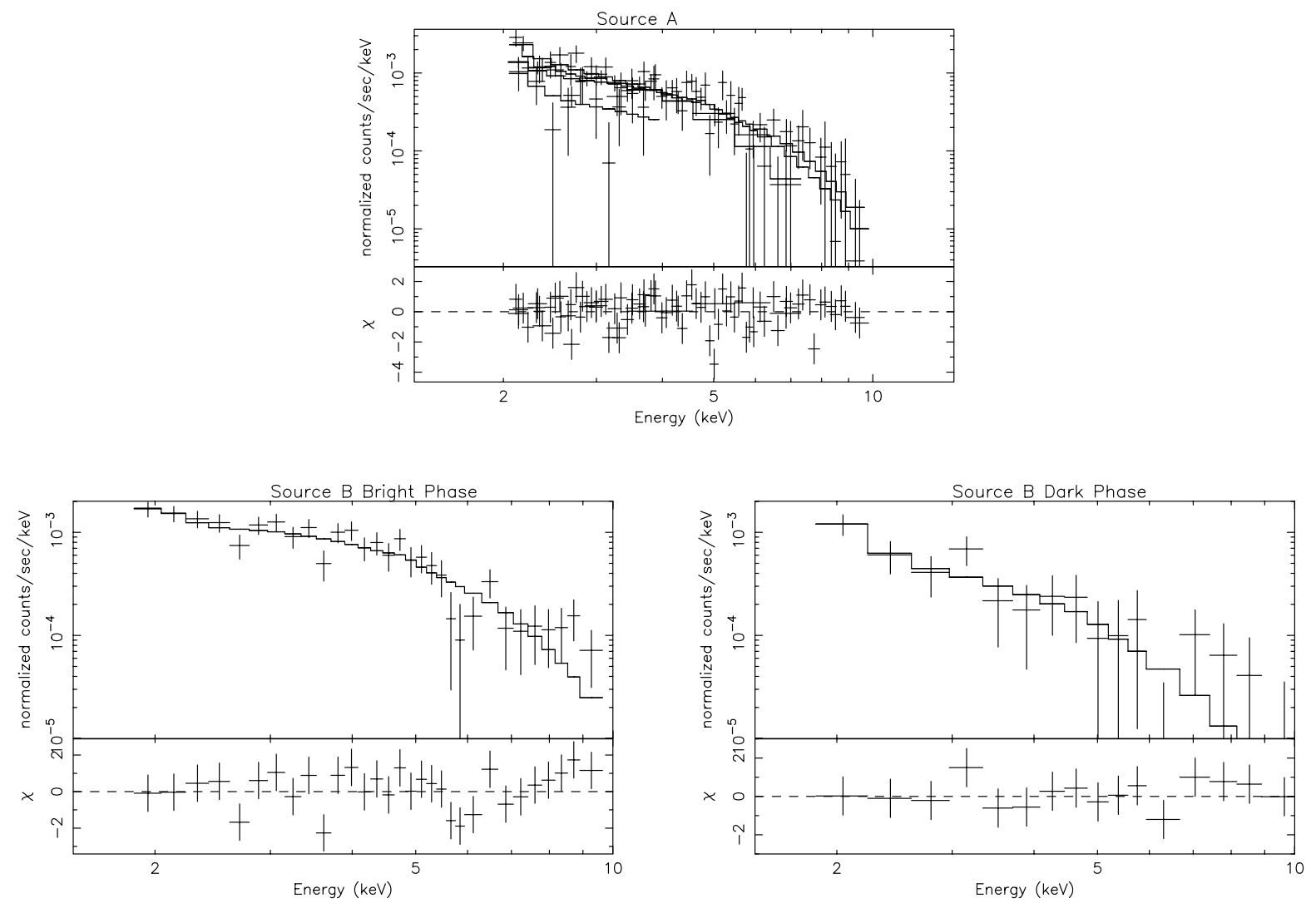

Fig. 8. X-ray spectra of the source A and B. a) ASCA GIS/SIS (1993) and BeppoSAX LECS/MECS (2000) spectra of the source A. b) MECS spectra of the source B. The left and right are obtained in the bright and faint phase, respectively.

the PDS is also likely to come from M 51. In addition, there are no other bright X-ray point sources with a flux $>10^{-12} \mathrm{erg} \mathrm{s}^{-1} \mathrm{~cm}^{-2}(0.1-2 \mathrm{keV})$ within 2 degrees of the M 51 nucleus in the ROSAT PSPC image. Consequently, we conclude that the hard X-ray emission detected in the PDS is associated with the nucleus of M 51. Then, M 51 is found to be one of the brightest low luminosity AGNs in the sky above the $10 \mathrm{keV}$ band, together with M 81 and NGC 4258 (Pellegrini et al. 2000; Fiore et al. 2001). It is interesting that these two objects are not Compton thick, unlike M 51.

There still remains a difference between the Ginga and other satellite observations; the X-ray flux below $10 \mathrm{keV}$ obtained with Ginga was higher than that measured by other instruments by a factor of more than 20, and the X-ray spectrum obtained with Ginga exhibited no absorption features. One possibility is the change of the column density of the absorbing material in the line of sight; the absorption happened to be weak in the Ginga observation. Such a case is in fact observed for the famous Seyfert galaxy NGC 4051 (Uttley et al. 1999), and for NGC 1365 (Risaliti et al. 2000) with BeppoSAX, and thus such a behavior is not unique to M 51. Another scenario would have a large change in the soft $(<10 \mathrm{keV})$ flux, by a factor of more than 20. However, the X-ray flux above $10 \mathrm{keV}$ band is almost the same between the Ginga and BeppoSAX observation epochs, and in addition agrees with the prediction from $L_{\mathrm{OIII}}$ and $L_{\mathrm{H} \alpha}$, suggesting that the former explanation is more likely.

\subsection{A neutral Fe $K$ line and reflected continuum}

BeppoSAX detected neutral $\mathrm{Fe} K$ lines from the M 51 nucleus, confirming the previous ASCA detection (Terashima et al. 1998a). This line is likely to arise via fluorescence from neutral material. The equivalent width of the $\mathrm{Fe} \mathrm{K}$ line, $0.9_{-0.5}^{+0.4} \mathrm{keV}$ is consistent with the ASCA 1993 result (Terashima et al. 1998a). The equivalent width of the Fe $\mathrm{K}$ line can be explained as arising via reflection by the reprocessing material covering $\sim 2 \pi$ around the nucleus with $1 \times$ Solar metal abundances. Other wellknown examples of objects indicating such geometry are NGC 6552 (Fukazawa et al. 1994) and Circinus Galaxy (Matt et al. 1996).

A natural association of the Fe $\mathrm{K}$ line would be with the dense, neutral material present around the nucleus; such material is most likely responsible for the Compton reflection component, seen in many Seyfert 1 galaxies. We investigated how strong of such reflection component is in the BeppoSAX data, but obtained a small upper limit corresponding to a solid angle subtended by the reflector of $18 \%$ of $2 \pi$. If the material responsible for the Fe K 
Table 2. Results of spectral fittings of source A and B with various models above $2 \mathrm{keV}$.

\begin{tabular}{|c|c|c|c|c|c|c|}
\hline $\begin{array}{c}k T^{r} \\
(\mathrm{keV}) \\
\end{array}$ & $\begin{array}{c}\text { norm }^{r} \\
\left(10^{11} \mathrm{~cm}^{-5}\right)\end{array}$ & $\overline{\alpha_{\mathrm{ph}}^{p}}$ & norm $^{p}$ & $\begin{array}{c}k T_{\text {in }}^{d} \\
(\mathrm{keV})\end{array}$ & $\begin{array}{c}\text { norm }^{d} \\
10^{-3} \\
\end{array}$ & $\chi^{2} /$ dof \\
\hline \multicolumn{7}{|c|}{ Source A: GIS + SIS + LECS + MECS } \\
\hline \multicolumn{7}{|c|}{ Power law Model } \\
\hline 0.39 Fix & $0.4_{-0.4}^{+4.6}$ & $2.19_{-0.35}^{+0.25}$ & $1.77_{-0.73}^{+0.64}$ & & & 1.01 \\
\hline \multicolumn{7}{|c|}{ MCD Model } \\
\hline 0.39 Fix & $6.0_{-3.6}^{+3.5}$ & & & $1.80_{-0.31}^{+0.44}$ & $2.0_{-1.1}^{2.5}$ & 1.00 \\
\hline \multicolumn{7}{|c|}{ Source B bright phase: LECS + MECS } \\
\hline \multicolumn{7}{|c|}{ Power law Model } \\
\hline 0.35 Fix & $0.21_{-0.19}^{+4.18}$ & $2.10 \pm 0.17$ & $1.78_{-0.37}^{+0.42}$ & & & 1.04 \\
\hline \multicolumn{7}{|c|}{ MCD Model } \\
\hline 0.35 Fix & $0.53_{-0.16}^{+0.18}$ & & & $1.50_{-0.18}^{+0.21}$ & $5.3_{-2.1}^{+3.0}$ & 1.12 \\
\hline \multicolumn{7}{|c|}{ Source B faint phase: LECS + MECS } \\
\hline \multicolumn{7}{|c|}{ Power law Model } \\
\hline 0.35 Fix & $<0.33$ & $3.09_{-0.41}^{+0.31}$ & $2.36_{-0.86}^{+0.65}$ & & & 0.39 \\
\hline \multicolumn{7}{|c|}{ MCD Model } \\
\hline 0.35 Fix & $0.40_{-0.32}^{+0.30}$ & & & $0.70_{-0.19}^{+0.29}$ & $73_{-60}^{+333}$ & 0.51 \\
\hline \multicolumn{7}{|c|}{$r:$ parameters of Raymond-Smith model. } \\
\hline \multicolumn{7}{|c|}{$p$ : parameters of Power law model. } \\
\hline \multicolumn{7}{|c|}{$d:$ parameters of MCD model. } \\
\hline \multicolumn{7}{|c|}{ The unit of norm ${ }^{p}$ is $10^{-3} \mathrm{c} \mathrm{s}^{-1} \mathrm{~cm}^{-2} \mathrm{keV}^{-1}$ at $1 \mathrm{keV}$. } \\
\hline \multicolumn{7}{|c|}{$\begin{array}{l}\text { The norm }{ }^{d} \text { represents }((\operatorname{Rin} / 1 \mathrm{~km}) /(D / 10 \mathrm{kpc}))^{2} \cos \theta \text { where } \operatorname{Rin} \text { and } \theta \text { are an inner radius } \\
\text { and an inclination angle of the disk, respectively, and } D \text { is a distance to the source. }\end{array}$} \\
\hline
\end{tabular}

Table 3. ROSAT HRI Flux of the Source B and C.

\begin{tabular}{cccccc}
\hline \hline Time & 1991.12 .07 & 1992.01 .08 & $1994.05 .22-23$ & $1994.06 .18-24$ & $1997.12 .26-30$ \\
& & & & \\
\multicolumn{7}{c}{$1992.05 .22-25$} \\
1992.06 .05 & & & \\
\hline exposure & $16097 \mathrm{~s}$ & $10411 \mathrm{~s}$ & $9392 \mathrm{~s}$ & $36343 \mathrm{~s}$ & $8104 \mathrm{~s}$ \\
$\mathrm{~B}$ & 1.5 & 1.2 & 1.8 & 2.1 & 0.9 \\
$\mathrm{C}$ & 2.2 & $<1.0$ & 2.4 & 1.7 & $<0.4$ \\
\hline
\end{tabular}

The unit is $10^{-13} \mathrm{erg} \mathrm{s}^{-1} \mathrm{~cm}^{-2}$. The conversion from counts to source flux density is $4.08 \times 10^{-11} \mathrm{erg} \mathrm{cm}^{-2}$ count $^{-1}$, from Ehle et al. (1995). The data in 1992 are quoted from Ehle et al. (1995).

line and the Compton reflection were to be the same, this value seems to be in contradiction with the Fe $\mathrm{K}$ equivalent width. A more likely scenario is that the Fe $\mathrm{K}$ line is produced in a Compton-thick region in transmission. In fact, the equivalent width of the $\mathrm{Fe} \mathrm{K}$ line can be reproduced by such a situation with a column density of $\sim 10^{24} \mathrm{~cm}^{2}$, covering factor of $2 \pi$, and a Solar Fe abundance (Makishima 1986).

\subsection{Time variability}

The bright hard component that was detected with Ginga and BeppoSAX, presumably due to direct nuclear emission, has almost constant flux during the BeppoSAX observation. Such a trend of a weak time variability is similar to that seen in other LLAGNs (Ishisaki et al. 1996; Iyomoto et al. 1996, 2001; Iyomoto 1999;
Reynolds et al. 2000), and is not consistent with the trend seen in Seyfert galaxies (Nandra et al. 1997; Hayashida et al. 1998). We note that a scenario involving an advection dominated accretion flow (ADAF) is consistent with such a behavior, but any more detailed discussion of the applicability of ADAFs to LLAGN is beyond the scope of this paper (but see, e.g., Lasota et al. 1996).

On the other hand, we detected time variability of two quantities: the weak hard X-ray emission below $10 \mathrm{keV}$, and the absorption column density for direct nuclear emission. These are indicative of a change of conditions of the material surrounding the nucleus. The former, which is thought to be a scattered or reflected nuclear component, exhibits a long time variability, by a factor of $2-3$ on a time scale of years, through ASCA and BeppoSAX observations (Fig. 7). The strength of the Fe $\mathrm{K}$ line also varied simultaneously with the continuum by almost the same factor. 
Regarding the latter, we measure the intrinsic absorption column density changes by a factor of $>100$ within several years between the Ginga and ASCA observations. These indicate that the Compton-thick absorption material has a spatial scale (meaning the size and/or a distance from the nucleus) of a parsec. Such a parsec - scale torus material is also suggested by other observations (Greenhill et al. 1996; Gallimore et al. 1997; Siebenmorgen et al. 1997; Matt 2000). Further variability studies of direct nuclear emission on shorter time scales at energies above $10 \mathrm{keV}$, such as with INTEGRAL and Astro-EII HXD, are necessary to characterize the dimensions of this component in more detail.

\subsection{Obscured low luminosity AGNs}

ASCA survey showed that LINER galaxies often exhibit hard X-ray emission with X-ray luminosity of $(2-5) \times$ $10^{40} \mathrm{erg} / \mathrm{s}(2-10 \mathrm{keV})$ and power law spectra with photon indices of $\sim 1.7$ (Makishima et al. 1994; Ishisaki et al. 1996; Iyomoto et al. 1996; 1997; 1998; Terashima et al. 1998a; 1998b; 2000). However, there are LINERs which do not show a significant excess of hard X-ray emission below $10 \mathrm{keV}$. Interestingly, ASCA results show that some LLAGNs also exhibit an intrinsic absorption of $\sim 10^{23} \mathrm{~cm}^{-2}$ which is comparable to Comptonthin Seyfert 2 galaxies. Furthermore, ASCA found two LLAGNs, M 51 and NGC 1365 (Terashima et al. 1998a; Iyomoto et al. 1997), which exhibit a flat hard X-ray component with a strong neutral Fe $\mathrm{K}$ line, and thus these are good candidates for the low luminosity Compton-thick Seyfert 2 galaxies. In this context, our BeppoSAX observations confirm M 51 as a Compton-thick LLAGN. These results suggest that the distribution of the absorption column density of LLAGNs may be similar to that of Seyfert galaxies, and thus a significant fraction of LINERs may be also completely obscured below $10 \mathrm{keV}$, as is the case for Seyfert galaxies. LLAGNs are thought to be quite numerous because LINERs are quite numerous (Keel 1983; Ho et al. 1997a). With this, future X-ray observations of LINER galaxies may reveal many LLAGNs in galaxies that are considered otherwise "normal". Understanding the relationship between LINERs and LLAGN is likely to be an important step in understanding the link between evolution of normal and active galaxies.

\subsection{Ultra-luminous off-nuclear $X$-ray sources}

M 51 contains several ultra-luminous off-nuclear X-ray sources found with Einstein and ROSAT (Palumbo et al. 1985; Marston et al. 1995; Ehle et al. 1995). ASCA and BeppoSAX observations resolved three of them, and all three exhibit time variability of a factor of $\sim 3$. The X-ray luminosity measured in those sources $-10^{39-40} \mathrm{erg} \mathrm{s}^{-1}$ cannot be explained by neutron star binaries, as the Eddington limit for those is $2 \times 10^{38} \mathrm{erg} \mathrm{s}^{-1}$. The X-ray spectra for two of them can be fitted with power law or disk blackbody models. This spectral shape is similar to that seen in other ultra-luminous off-nuclear X-ray sources in other galaxies (Mizuno et al. 1999; Makishima et al. 2000; Mizuno 2000). These bright X-ray sources in nearby galaxies are postulated to be black hole binaries, where spectra (and in particular, the disk black body temperature and normalization) imply Kerr black holes with masses $\sim 100 M_{\odot}$ (Mizuno et al. 1999; Makishima et al. 2000; Mizuno 2000). We suggest that the bright, offnuclear X-ray sources in M 51 detected with ASCA and BeppoSAX are objects of the same type. The strong variability of these sources is similar to that often observed in the Galactic black hole candidates. Since their X-ray spectra were obtained only in their bright states, it would be quite interesting to know what are the X-ray spectra in their faint states, and in particular, whether they exhibit hard power law spectra in their faint states, similar to the Galactic black hole candidates. So far, two ultraluminous off nuclear X-ray sources in IC 342 exhibited such a spectral transition (Kubota et al. 2001), suggesting that these are black-hole binaries. Since it contains relatively many ultra-luminous off-nuclear X-ray sources, M 51 is an interesting target for repeated observations by Chandra and XMM-Newton to study such sources.

Acknowledgements. We are grateful to Prof. Greg Madejski for correction of the English in the manuscript. The authors also thank the anonymous referee for careful reading and helpful comments, and thank as well operation and calibration teams of BeppoSAX and ASCA.

\section{References}

Anders, E., \& Grevesse, N. 1989, Geochim. Cosmochim. Acta., 53, 197

Boella, G., Butler, R., Perola, G. C., et al. 1997a, A\&AS, 112, 299

Boella, G., Chiappetti, L., Conti, G., et al. 1997b, A\&AS, 112, 372

Cappi, M., Bassani, L., Comastri, A., et al. 1999, A\&A, 344, 857

Done, C., Madejski, G. M., \& Smith, D. A. 1996, ApJ, 463, L63

Ehle, M., Pietsch, W., \& Beck, R. 1995, A\&A, 295, 289

Filippenko, A. V., \& Sargent, W. L. W. 1985, ApJS, 57, 503

Fiore, F., Guainazzi, M., \& Grandi, P. 1999, Cookbook for BeppoSAX NFI Spectral Analysis

Fiore, F., Pellegrini, S., Matt, G., et al. 2001 [astro-ph/0102438]

Frontera, F., Costa, E., Dal Fiume, D., et al. 1997, A\&AS, 112, 357

Fukazawa, Y., Makishima, K., Ebisawa, K., et al. 1994, PASJ, 46, L141

Gallimore, J. F., Baum, S. A., \& O'Dea, C. P. 1997, Nature, 388,852

Greenhill, L. J., Gwinn, C. R., Antonucci, R., \& Barvainis, R. 1996, ApJ, 472, L21

Hayashida, K., Miyamoto, S., Kitamoto, S., et al. 1998, ApJ, 500,642

Ho, L. C., Filippenko, A. V., \& Sargent, W. L. W. 1997a, ApJ, 487, 568 
Ho, L. C., Filippenko, A. V., \& Sargent, W. L. W. 1997b, ApJS, 112,315

Ho, L. C., Filippenko, A. V., Sargent, W. L. W., \& Peng, C. Y. 1997c, ApJS, 112, 391

Immler, S., Pietsch, W., \& Aschenbach, B. 1998, A\&A, 336, L1

Ishisaki, Y., Makishima, K., Iyomoto, N., et al. 1996, PASJ, 48,237

Iyomoto, N., Makishima, K., Fukazawa, Y., et al. 1996, PASJ, 48,231

Iyomoto, N., Makishima, K., Fukazawa, Y., et al. 1997, PASJ, 49,425

Iyomoto, N., Makishima, K., Matsushita, K., et al. 1998, ApJ, 503,168

Iyomoto, N. 1999, Ph.D. Thesis, University of Tokyo

Iyomoto, N., \& Makishima, K. 2001, MNRAS, 321, 767

Keel, W. C. 1983, ApJ, 269, 466

Kohno, K., Kawabe, R., Tosaki, T., \& Okumura, S. K. 1996, ApJ, 461, L29

Kotoku, J., Mizuno, T., Kubota, A., \& Makishima, K. 2001, PASJ, 52, 1081

Kubota, A., Mizuno, T., Makishima, K., et al. 2001, ApJ, 547, L119

Lasota, J.-P., Abramowicz, M. A., Chen, X., et al. 1996, ApJ, 462,142

Maiolino, R., Krabbe, A., Thatte, N., \& Genzel, R. 1998, ApJ, 493, 650

Makishima, K. 1986, The Physics of Accretion onto Compact Objects, Lect. Notes Phys. 266, ed. K. P. Mason, M. G. Watson, \& N. E. White (Springer-Verlag, Berlin Heidelberg New York), 249

Makishima, K., Maejima, Y., Mitsuda, K., et al. 1986, ApJ, 308,635

Makishima, K., Ohashi, T., Kondo, H., et al. 1990, ApJ, 365, 159

Makishima, K., Fujimoto, R., Ishisaki, Y., et al. 1994, PASJ, 46, L77

Makishima, K., Tashiro, M., Ebisawa, K., et al. 1996, PASJ, 48,171

Makishima, K., Kubota, A., Mizuno, T., et al. 2000, ApJ, 535, 632

Marston, A. P., Elmegreen, D., Elmegreen, B., et al., 1995, ApJ, 438, 663

Matsumoto, H., \& Tsuru, T. G. 1999, PASJ, 51, 321

Matt, G., Fiore, F., Perola, G. C., et al. 1996, MNRAS, 281 , L69
Matt, G., Guainazzi, M., Maiolino, R., et al. 1999, A\&A, 341, L39

Matt, G. 2000, A\&A, 35, L31

Mitsuda, K., Inoue, H., Koyama, K., et al. 1984, PASJ, 36, 741

Mizuno, T., Ohnishi, T., Kubota, A., et al. 1999, PASJ, 51, 663

Mizuno, T. 2000, Ph.D. Thesis, University of Tokyo

Mulchaey, J. S., Koratkar, A., Ward, M. J., et al. 1994, ApJ, 436,586

Nandra, K., George, I. M., Mushotzky, R. F., et al. 1997, ApJ, 477,602

Ohashi, T., Ebisawa, K., Fukazawa, Y., et al. 1996, PASJ, 48, 157

Okada, K., Dotani, T., Makishima, K., et al. 1998, PASJ, 50, 25

Palumbo, G. G. C., Fabbiano, G., Trinchieri, G., Fransson, C. 1985, ApJ, 298, 259

Parmar, A. N., Martin, D. D. E., Bavdaz, M., et al. 1997, A\&AS, 112, 309

Pellegrini, S., Cappi, M., Bassani, L., et al. 2000, A\&A, 353, 457

Ptak, A., Serlemitsos, P., Yaqoob, T., \& Mushotzky, R. F. 1999, ApJS, 120, 179

Raymond, J. C., \& Smith, B. W. 1977, ApJS, 35, 419

Reynolds, C. S., Nowak, M. A., \& Maloney, P. R. 2000, ApJ, 540,143

Risaliti, G., Maiolino, R., \& Salvati, M. 1999, ApJ, 522, 157

Risaliti, G., Maiolino, R., \& Bassani, L. 2000, A\&A, 356, 33

Sandage, A., \& Tammann, G. A. 1975, ApJ, 196, 313

Siebenmorgen, R., Moorwood, A., Freudling, W., et al. 1997, A\&A, 325, 450

Stark, A. A., Gammie, C. F., Wilson, R. W., et al. 1992, ApJS, 79,77

Stauffer, J. R. 1982, ApJ, 262, 66

Tanaka, Y., Inoue, H., \& Holt, S. S. 1994, PASJ, 46, L37

Terashima, Y., Ptak, A., Fujimoto, R., et al. 1998a, ApJ, 496, 210

Terashima, Y., Kunieda, H., Misaki, K., et al. 1998b, ApJ, 503, 212

Terashima, Y., Ho, L. C., \& Ptak, A. F. 2000, ApJ, 539, 161

Terashima, Y., \& Wilson, A. S. 2001, [astro-ph/0103287]

Uttley, P., McHardy, I. M., Papadakis, I. E., et al. 1999, MNRAS, 307, L6

Ward, M. J., Done, C., Fabian, A. C., et al. 1988, ApJ, 324, 767 